\title{
Motion Characteristics of FLNG Systems under the Effect of Drift Motions of Two Floating Bodies
}

\author{
Qiao Lia, Kohei Sugata ${ }^{a^{*}}$, Yasunori Niheia, Yoshiho Ikedaa \\ ${ }^{a}$ Department of Marine System Engineering, Osaka Prefecture University, Sakai, Japan \\ *Corresponding author: ri_k@marine.osakafu-u.ac.jp
}

Article history

Received :2 July2013

Received in revised form

5 November 2013

Accepted :25 November 2013

Graphical abstract

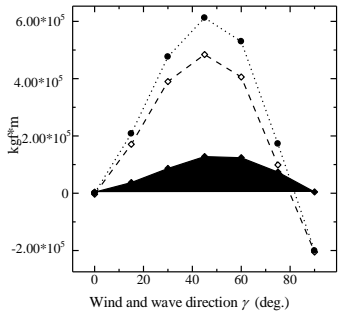

\section{Abstract}

In recent years, some small and midsize gas fields are being developed all over the world, and FLNG systems which include LNG FPSO and shuttle LNG tank are going to be used. In rough sea, when produced LNG is offloaded from the FPSO to the shuttle tanker, two ships will be moored. In this study, we are focusing on characteristics of FLNG systems in waves and wind. Experimental studies were performedto observe motion of two floating bodies in tandem condition in regular waves. In the tank test, we observed drastic yawing and drift motions of a shuttle LNG tanker. These phenomena might be a threatto the safety of a FLNG system. In this paper, we will reveal a cause of these phenomena and evaluate a FLNG system by using a simple method.

Keywords: LNG FPSO; shuttle LNG tanker; drift motion; mooring; tandem condition

(C) 2014 Penerbit UTM Press. All rights reserved

\subsection{INTRODUCTION}

Recently, people all over the world are concerned with problems of greenhouse effect on the earth. Rising populations also reads to a lack of energy all over the world. Natural gas exists all over the world. Furthermore, Carbon dioxide emission produced by the use of natural gas is less than the produced $\mathrm{CO}_{2}$ when using oil. So we should take an initiative in using natural gas in the future.

The FLNG systems are going to be used in natural gas mined, when natural gas fields at deep sea, especially small and midsize gas fields are developed. LNG FPSO can produce,storage and offload natural gas at deep sea. Currently it is assumed that theFLNG systems will be used in calm sea. However, these systems might be used in rough sea in the future.

In rough sea, two floating bodies will be moored in tandem when produced LNG is offloaded from the FPSO to the shuttle LNG tanker.However, there are concerns about offloading mechanism, cut operating rates of offloading LNG and safety of the system in tandem condition. So we are focusing on the motion characteristics of a FLNG system that two floating bodies are in tandem condition in waves and wind. The experimental studies are performedto observe motion of two floating bodies in tandem condition in regular waves.In the tank test, we observedrastic yawing and drift motions of a shuttle tanker. These phenomena might be a threatto the safety of the FLNG system.In this paper, we will reveal a cause of these phenomena and evaluate a FLNG system by using a simple method.

\subsection{EXPERIMENTS}

\subsection{Outline of a Tank Rest}

It is necessary that FLNG systems can produce LNG even in rough sea. In the rough sea, drift motion and drastic ship motions might be occurred. So we conduct the experimental tests to measure ship motions of the LNG FPSO and the shuttle tanker. The principal perpendiculars of the LNG FPSO and the shuttle tanker are shown in Table 1 and the body plans of the LNG FPSO and the shuttle tanker are also shown in Figure 1 and Figure 2.

Table 1 The principal particulars of two ships

\begin{tabular}{|c|c|c|c|c|}
\hline \multirow{2}{*}{} & \multicolumn{2}{|c|}{ LNG FPSO } & \multicolumn{2}{c|}{ Shattle tanker } \\
\cline { 2 - 5 } & Full scale & model & Full scale & model \\
\hline \hline Loa $(\mathrm{m})$ & 302 & 2.00 & 280 & 1.86 \\
\hline $\mathrm{Lpp}(\mathrm{m})$ & 302 & 2.00 & 272 & 1.80 \\
\hline $\mathrm{B}(\mathrm{m})$ & 60 & 0.397 & 43.5 & 0.288 \\
\hline $\mathrm{d}(\mathrm{m})$ & 10.0 & 0.066 & 8.50 & 0.056 \\
\hline $\mathrm{D}(\mathrm{m})$ & 33.0 & 0.218 & 26.0 & 0.172 \\
\hline Displacement(kg) & $1.81^{*} 10^{\wedge} 8$ & 52.4 & $7.46^{*} 10^{\wedge} 7$ & 21.6 \\
\hline $\mathrm{GM}(\mathrm{m})$ & 20.6 & 0.137 & 6.63 & 0.0440 \\
\hline kyy/Lpp & 0.222 & 0.222 & 0.281 & 0.281 \\
\hline Natural period of Roll $(\mathrm{s})$ & 12.3 & 1.00 & 10.9 & 0.889 \\
\hline
\end{tabular}




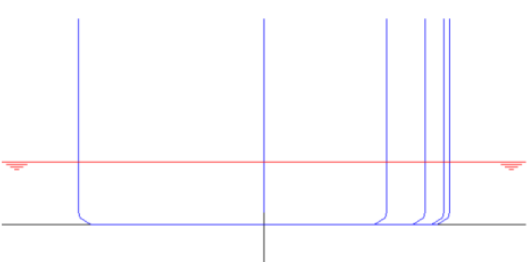

Figure 1 The body plan of the LNG FPSO

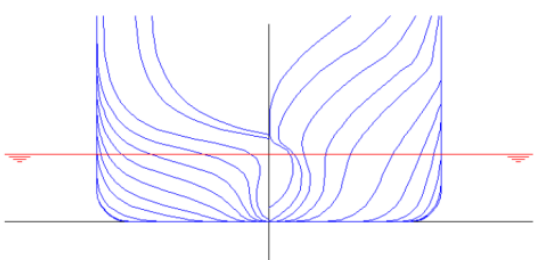

Figure 2 The body plan of the shuttle tanker

\subsection{Experimental Conditions of the Tank Test}

Table 2 shows the experimental conditions in tandem condition. Figure 3 also shows the experimental coordinate systems with their originatthe center of gravity of two floating bodies. Draft of the LNG FPSO is set $0.066 \mathrm{~m}$ and draft of the shuttle tanker is set $0.056 \mathrm{~m}$ in model scale. Each model ship is connected by the linear spring. The coefficient of this spring is $0.014 \mathrm{~N} / \mathrm{mm}$ in model scale

Table 2 The experimental conditionsfor the tandem condition

\begin{tabular}{c|ccccc}
\hline Wave direction $\beta$ (deg.) & & & 180 & & \\
\hline \hline Wave period $T_{w}(\mathrm{~s})$ & 0.6 & 0.7 & 1.0 & 1.2 & 1.6 \\
\hline Wave height $H_{w}(\mathrm{~m})$ & & 20 & & 40 & \\
\hline
\end{tabular}

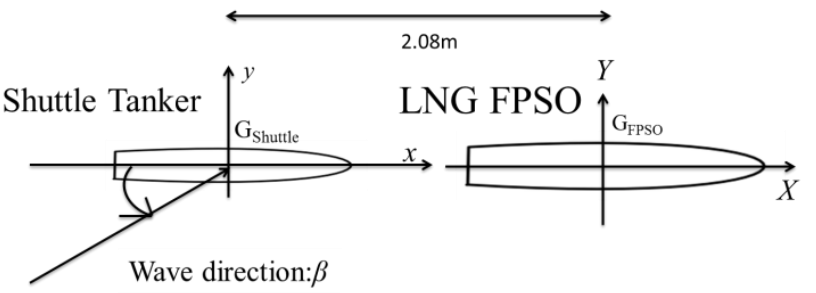

Figure 3 An experimental coordinate system

\subsection{Results of the Tank Test}

The observed viewof the tank tests were summarized in this section. We conducted experiments on the ship motion of the LNG FPSO and the shuttle tanker in the regular waves. As shown in Figure 4, shuttle tanker moved in the yawing direction drastically, when the wave period is 1.0 second in the tandem condition. If these phenomena will occur in real sea, the distance between two ships ismuch closer and risk of crash between two floating bodies might be increased. These phenomena also might decrease in operating rates of the offloading.

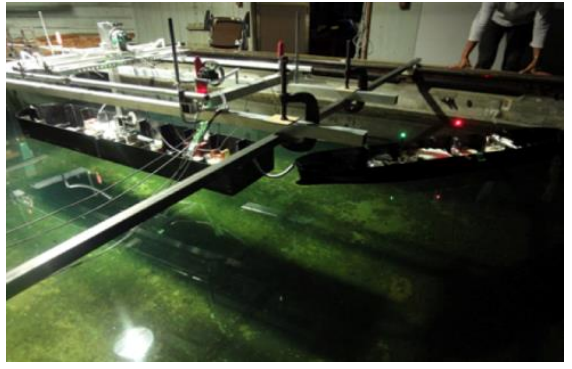

Figure 4 Drastic yawing motion in tandem condition

\subsection{INVESTIGATION OF THE CAUSE OF DRASTIC YAWING MOTION}

In the experiments, the shuttle LNG tanker moved in the yawing direction drastically. So we will investigatethe cause and provide qualitative analysis of these phenomena.

3.1 Mean Wave Drift Forces and Mean Wave Drift Moments Acting on the Shuttle Tanker in Regular Waves

Newman (1967) calculated drift forces and drift moments acting on a rectangular object and Series 60 (Block coefficient $C_{b}$ is $0.60)$. These results, especially the rectangular object, were revealed that the floating body would increase the yawing angle in the wave drift moments. That is, it is assumed that a shuttle tanker might be slued by the wave drift moment when a bow of the ship yaws relative to the wave direction.

\subsection{WAVE DRIFT FORCES AND DRIFT MOMENT}

\subsection{Theoretical Approach of Mean Wave Drift Forces and} Mean Wave Drift Moments

Mean wave drift forces and mean wave drift moments in regular waves acting on the floating body are calculated by means of the momentum theory propounded by Maruo (1960) and Newman (1967). z-up cylindrical coordinate system with their origin at the center of gravity of the floating body are used. Mean wave drift forces and mean wave drift moments are written by Equations (1)(3).

$$
\begin{gathered}
F_{x}=\iint_{S_{\infty}}\left(P \cos \theta+\rho U_{r}\left(U_{r} \cos \theta-U_{\theta} \sin \theta\right)\right) r d \theta d z \\
F_{y}=\iint_{S_{\infty}}\left(P \sin \theta+\rho U_{r}\left(U_{r} \sin \theta+U_{\theta} \cos \theta\right)\right) r d \theta d z \\
M_{z}=\rho \iint_{S_{\infty}} U_{r} U_{\theta} r^{2} d \theta d z
\end{gathered}
$$

where $P, \rho, r, U_{r}$, and $U_{\theta}$ are the water pressure, the fluid density, the radius of the circular cylinder, the current speed in the radial direction and the current speed in the circumferential direction. Considering the finite water depth $(z=-h)$, the incident wave potential $\phi^{(1)}$ and the disturbance potential due to the floating body $\phi_{B}$ are expressed in Equation 4 and Equation 5.

$$
\begin{gathered}
\phi^{(1)}=-\frac{i g A}{\omega} \frac{\cosh k(z+h)}{\cosh k h} \sqrt{\frac{2}{\pi k r}} \exp (i k r \cos \theta) \\
\phi_{B}=-\frac{i g A}{\omega} \frac{\cosh k(z+h)}{\cosh k h} H(\theta) \sqrt{\frac{2}{\pi k r}} \exp (i k r-i(\pi / 4))
\end{gathered}
$$


By substituting Equation 4 and Equation 5 into the Equations 1-3, we obtain Equation 6-8.

$$
\begin{gathered}
F_{x}=\frac{\rho g A}{k} \frac{C_{g}}{C} \frac{1}{\pi} \int_{0}^{2 \pi}|H(\theta)|^{2}(\cos \beta-\cos \theta) d \theta \\
F_{y}=\frac{\rho g A}{k} \frac{C_{g}}{C} \frac{1}{\pi} \int_{0}^{2 \pi}|H(\theta)|^{2}(\sin \beta-\sin \theta) d \theta \\
M_{z}=-\rho g A \frac{1}{k^{2}} \frac{C_{g}}{C} \operatorname{Im}\left[\left.\frac{d H^{*}}{d \theta}\right|_{\theta=\beta}+\frac{1}{\pi} \int_{0}^{2 \pi} H(\theta) \frac{d H(\theta)^{*}}{d \theta} d \theta\right]
\end{gathered}
$$

$H(\theta)$ is Kochin function. $C_{g}$ and $C$ are the group velocity and the phase velocity of waves. Considering deep water waves, the relation between $C_{g}$ and $C$ is expressed in Equation 9.

$$
C_{g}=\frac{C}{2}
$$

\subsection{Hybrid Method}

In order to calculate mean wave drift forces and mean wave drift moments, we use a "Hybrid method" (Nihei, Kinoshita, Bao, 2008). Hybrid method is a compositealgorism that is combined with eigenfunction expansion and boundary element method. This method also uses Rankine source as its Green function. As shown in Figure 5, an artificial cylinder with radius $r_{0}$ is arranged in calculating area. Hybrid method uses the boundary element method (B.E.M) in the inner region of an artificial circular cylinder and an eigen function expansion in outer region of an artificial cylinder.

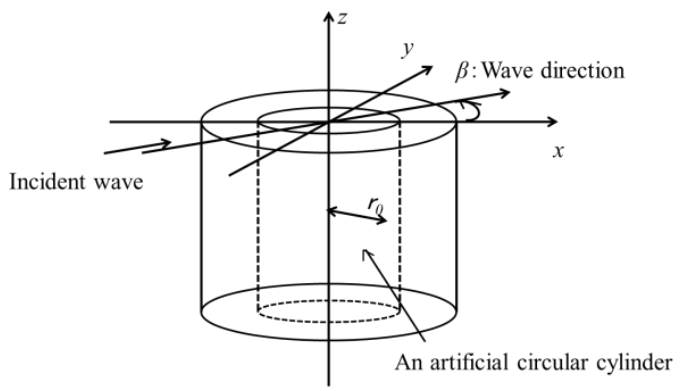

Figure 5 Coordinate systems for hybrid method function:

At first, scattering potential $\phi_{B}$ is expanded by means of eigen

$$
\phi_{B}=\sum_{m=0}^{\infty} \sum_{n=0}^{\infty} R_{m n}(r) Z_{m n}(z)\left(A_{m n}^{(1)} \cos n \theta+B_{m n}^{(1)} \sin n \theta\right)
$$

Where $A_{m n}{ }^{(l)}$ and $B_{m n}{ }^{(l)}$ are unknown quantity.

Furthermore, $R_{m n}(r)$ and $Z_{m n}(z)$ are eigen functions and are defined by Equation 11, and Equation 12.

$$
\begin{gathered}
R_{m n}(r)= \begin{cases}\frac{K_{n}\left(k_{m} r\right)}{K_{n}\left(k_{m} r_{0}\right)}, & m=0 \\
\frac{H_{n}\left(k_{0} r\right)}{H_{n}\left(k_{0} r_{0}\right)}, & m>0\end{cases} \\
Z_{m}(z)= \begin{cases}\frac{\cosh k_{0}(z+h)}{\cos \left(k_{0} h\right)}, & m=0 \\
\frac{\cosh k_{m}(z+h)}{\cos \left(k_{m} h\right)}, & m>0\end{cases}
\end{gathered}
$$

Where $H_{n}$ and $K_{n}$ are n-th Hankel function of the first kind and nth modified Bessel function in the second kind.

Secondly, we obtain an integral equation with a green function in the inner region of the artificial cylinder and eigenfunction on the artificial circular cylinder. Unknown potentials and coefficients $A^{(1)}{ }_{m n}, B^{(1)}{ }_{m n}$ are solved by means of Gaussian elimination for the simultaneous linear equations obtained from the boundary integral equation.

\subsection{Derivation of Kochin Function}

Scattering potential $\varphi_{B}$ is defined as Equation 10. Additionally, Scattering potential $\phi_{B}$ is expressed in Equation 13 when a radius of the artificial cylinder $r$ approaches infinityin terms of a definition of a Kochin function.

$\phi_{B}=\sum_{n=0}^{\infty} \sqrt{\frac{2}{\pi k r}} \frac{1}{H_{n}\left(k_{0} r_{0}\right)} e^{i k r-\frac{\pi}{4} i} e^{\frac{-n \pi}{2}} \frac{\cosh k(z+h)}{\cosh k h}\left(A_{0 n}^{(1)} \cos n \theta+B_{0 n}^{(1)} \operatorname{sinn} \theta\right)$

We use approximate expression of the 1st Hankel function at the infinite area.

Kochin function $H(\theta)$ is expressed in Equation 14 derived from Equation 5 and Equation 13.

$$
\begin{aligned}
& \left.H(\theta)=\sum_{n=0}^{\infty} \overline{\left(A_{0 n}^{(1)}\right.} \cos n \theta+\overline{B_{0 n}^{(1)}} \operatorname{sinn} \theta\right) \\
& \overline{A_{0 n}^{(1)}}=\frac{A_{0 n}^{(1)} e^{-\frac{n}{2} \pi}}{H_{n\left(k_{0} r_{0}\right)}\left(-\frac{i g A}{\omega}\right)} \\
& \overline{B_{0 n}^{(1)}}=\frac{B_{0 n}^{(1)} e^{-\frac{n}{2} \pi}}{H_{n\left(k_{0} r_{0}\right)}\left(-\frac{i g A}{\omega}\right)}
\end{aligned}
$$

Thus, the mean wave drift forces and mean wave drift moments are obtained by substituting Kochin function into Equation 6-8.

\subsection{Calculation Conditions}

The wave drift forces and the wave drift moments in regular waves are calculated under the following conditions. We use the modified Wigley model without bilge keels in the calculations.

$$
\begin{gathered}
\eta=\left(1-\xi^{2}\right)\left(1-\zeta^{2}\right)\left(1+a \xi^{2}\right)+b \zeta^{2}\left(1-\zeta^{8}\right)\left(1-\xi^{2}\right)^{4} \\
\xi=2 x / L, \eta=2 y / B, \zeta=z / d
\end{gathered}
$$

The hull shape whose parameters $a$ equal to 1 and $b$ equal to 0.2 in the Equation 15 is used. The wave frequency $\omega$ equal to 6.28 that is close to the natural circular frequency for the roll motion of the shuttle tanker.

The mesh model is shown in Figure 6 in these calculations. 320 computational meshes are set on a hull of the ship. A free surface is divided into 4 layers in the radius direction and 40 circumferences. An artificial boundary is divided into 6 sections in vertical direction and 40 circumferences. 


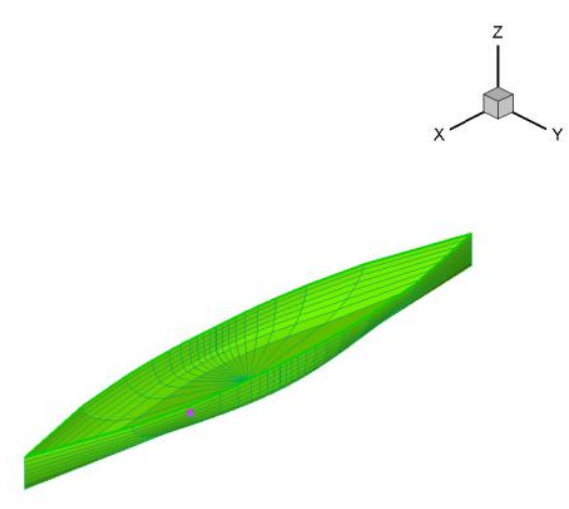

Figure 6 The mesh model of modified Wigley

4.5 Calculation Results of Mean Wave Drift Forces and Mean Wave Drift Moments in Regular Waves

Calculation results of mean wave drift forces and mean wave drift moments in case of the diffraction mode in regular waves (the wave frequency $\omega$ is $6.28 \mathrm{rad} / \mathrm{s}$ are shown in Figures 7-9. Each calculation result of wave drift forces and wave drift moments is non-dimensionalized in terms of the fluid density $\rho$, gravitational acceleration $g$, and wave amplitude $A$,and the length between perpendiculars $L_{p p}$.

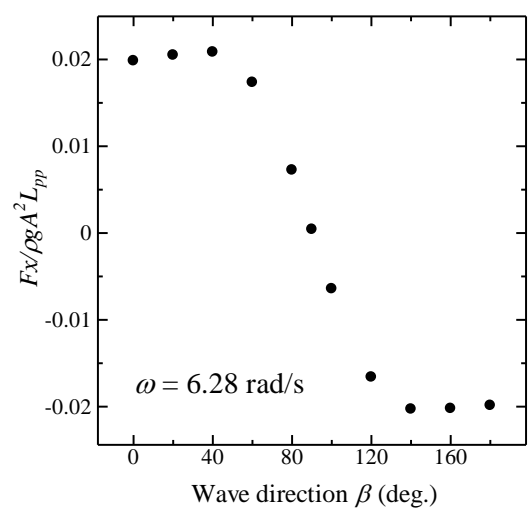

Figure 7 Calculation results of coefficients of mean wave drift forces in $\mathrm{x}$ - direction in regular waves (diffraction)

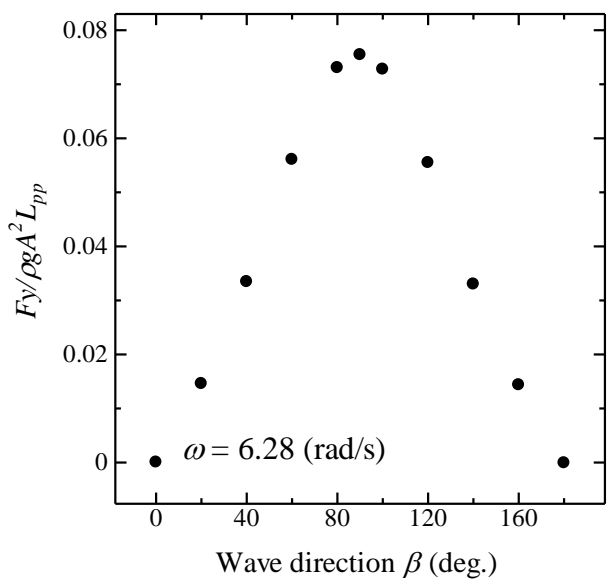

Figure 8 Calculation results of coefficients of mean wave drift forces in $y$-direction in regular waves (diffraction)

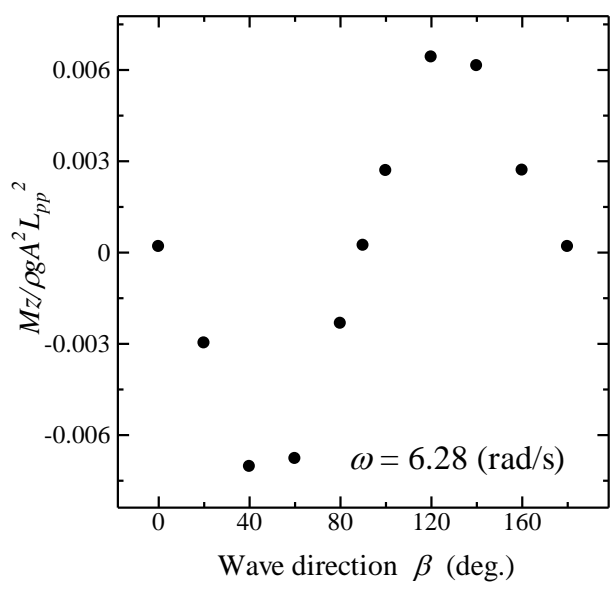

Figure 9 Calculation results of coefficients of mean wave drift moments in regular waves (diffraction)

As shown in Figure 9, drift moments are varied as a sine curve related to the wave direction. So it reveals that wave drift moments acting on the shuttle tanker generate the yawing motion when the bow of the shuttle tanker yaws relative to the wave direction.

\subsection{WIND FORCES AND WIND MOMENTS ACTING ON A SHUTTLE TANKER UNDER WIND SHIELDING EFFECT}

\subsection{Background of Wind Effect Acting on FLNG Systems}

In an operation of a FLNG system, wind effect largely affects each ship motion, production ratio of natural gas and so on. When the shuttle tanker is close to the LNG FPSO, for example, when produced LNG is offloaded from the LNG FPSO to the shuttle tanker, characteristics of wind forces and wind moments acting on two floating bodies are different from thoseof single body. Because of wind is blocked by the hull and structures on upper deck of the FPSO, when the FPSO is located in a windward side.This mutual interaction is called "wind shielding effect". In order to confirmcharacteristics of wind effects acting on the shuttle tanker under wind shielding effect, we measured the wind forces and moments acting on the shuttle tanker.

\subsection{Experimental Condition}

The wind forces and moments acting on the shuttle tanker were measured under the condition as shown in Table 3. As shown in Figure 10, we use a z-up system coordinates with their origin at midships of two floating bodies.The LNG FPSO and the shuttle tanker are set in the tandem, and the shuttle tanker is located on the leeside. The LNG FPSO is fixed in place. We measured wind forces in $x$-direction $F_{x w i n d}$, wind forces in $y$-direction $F_{y w i n d}$ and wind moments $M_{z \text { wind }}$.

Table 3 The experimental condition

\begin{tabular}{|c|c|c|c|c|c|}
\hline Wind direction $\chi$ (deg.) (FLNG) & \multicolumn{5}{|c|}{0} \\
\hline \multirow{2}{*}{ Wind direction $\gamma$ (deg.) (shuttle) } & 0 & 15 & 30 & 45 & 60 \\
\hline & & 75 & & 90 & \\
\hline$\rho$ wind $\left(\mathrm{kgf} / \mathrm{m}^{\wedge} 3\right)$ & \multicolumn{5}{|c|}{0.121} \\
\hline Wind speed $V$ wind $(\mathrm{m})$ & \multicolumn{5}{|c|}{3.8} \\
\hline
\end{tabular}




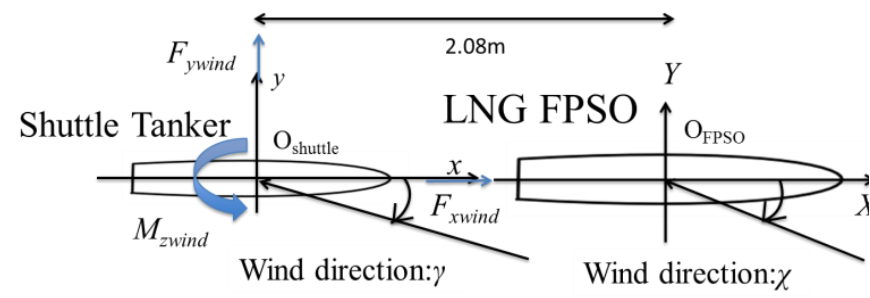

Figure 10 Coordinate systems for experiments
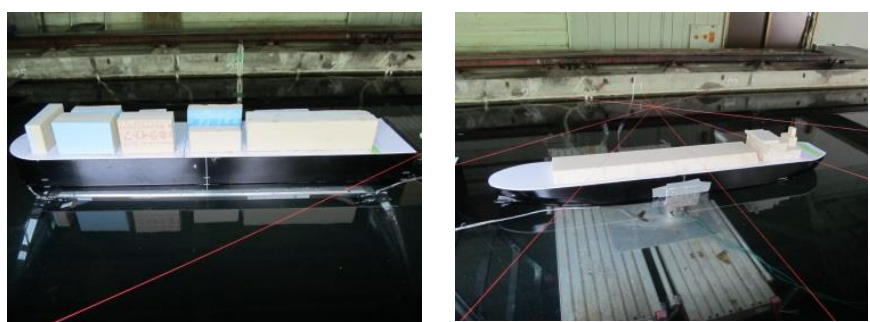

Figure 11 The hulls and structureson upper deck of two floating bodies. (Left: The LNG FPSO, Right: The shuttle tanker)

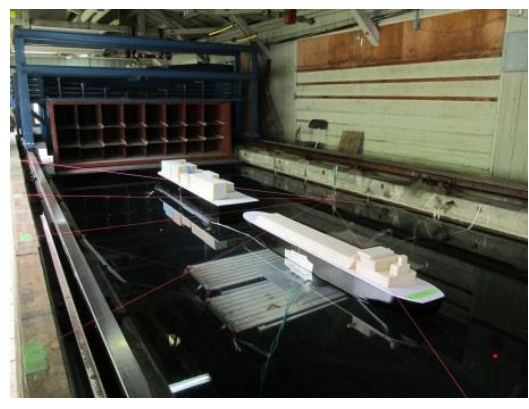

Figure 12 A photo of experiments

\subsection{Experimental Results of Wind Forces and Wind Moments Under Wind Shielding Effect}

Experimental results are shown in Figures 13-15. Each experimental results are non-dimensionalized in terms of the air density $\rho_{\text {air }}$, the frontal projected area $S_{f}$, the side projected area $S_{S}$, the wind velocity $V_{\text {wind }}$ and the length between perpendiculars $L_{p p}$.

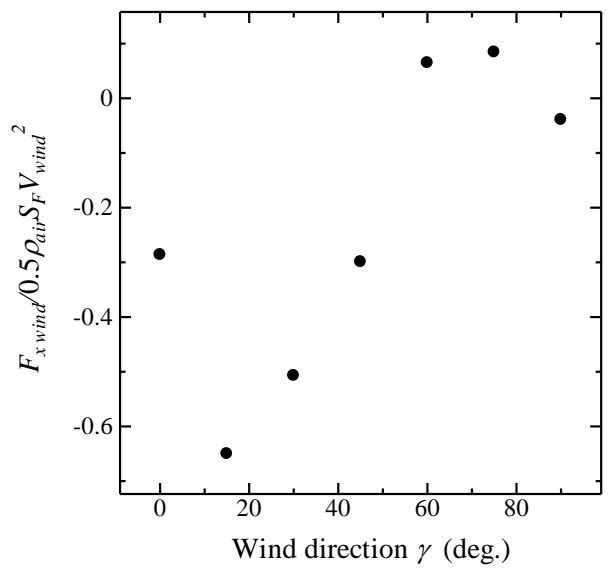

Figure 13 Experimental results of the wind forces in $\mathrm{x}$-direction acting on the shuttle tanker

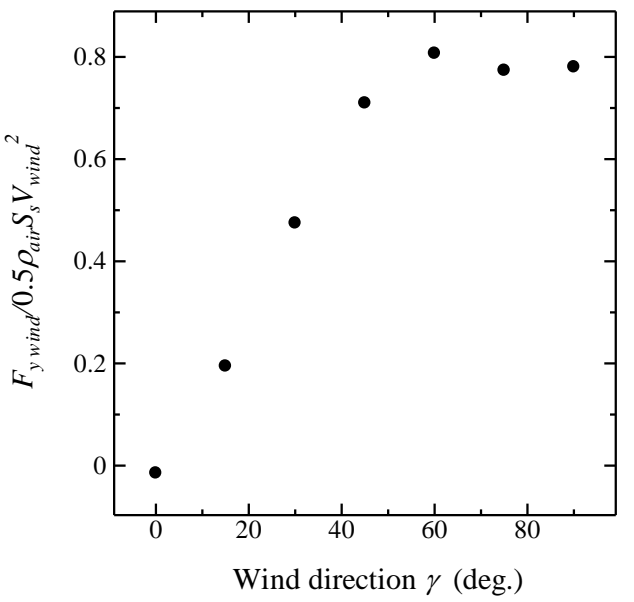

Figure 14 Experimental results of the wind forces in y-direction acting on the shuttle tanker

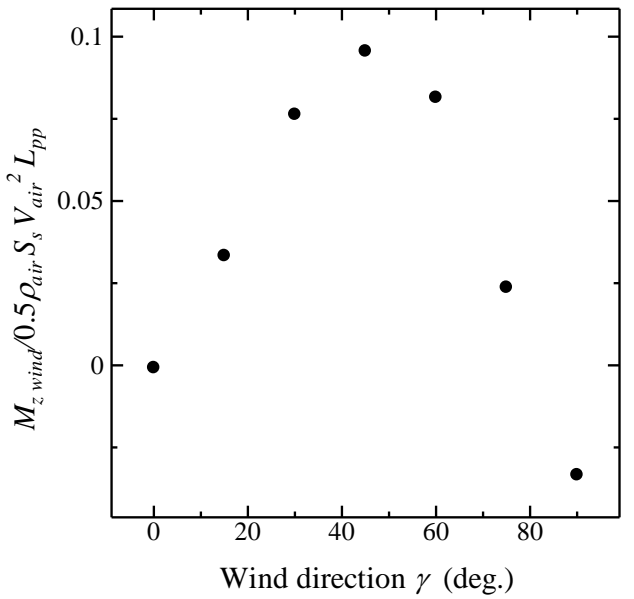

Figure 15 Experimental results of the wind moments acting on the shuttle tanker

As shown in Figure 15, the wind moments acting on the shuttle tanker is positive within a range from 0 degree to about 80 degrees. For this reason, it is revealed that point of application of wind moment is located forward to the midship of the shuttle tanker.

\subsection{MOMENTS IN THE WIND AND WAVE CONDITIONS UNDER TANDEM CONDITION}

In this section, yawing moments on the shuttle tanker in both regular waves and winds will be discussed. In the regular wave and wind condition, mean wave drift forces and wind forces act on the shuttle tanker. Current forces acting on the shuttle tanker is not considered. As simple method to estimate drift motion of the shuttle tanker in winds and waves, we investigate drift forces and moments acting on the shuttle tanker.

As shown in Figure 16, coordinate systemswith their origin at a center of gravity of two floating bodies. The LNG FPSO is fixed in place. For simplicity, the origins on each ship are not translated and do not rotate. Wind and wave are headed in the same direction 
The data of the wave drift forces and the wave drift moment shown in Figures 7-9 and wind forces and wind moments as shown in Figures 13-15 are used in investigation.

The wave drift forces and the wave drift moments in real scale under the condition of beaufort scale 4 are calculated. As for the calculation of the wave drift forces and the moment, the significant wave height under the condition of beaufort scale 4 (The kansai Society of Naval Architects, 1983)is employed in this paper. The wave amplitude $A$ is $0.5 \mathrm{~m}$, and the wave frequency $\omega$ is $6.28 \mathrm{rad} / \mathrm{s}$. The wind speed $V_{\text {wind }}$ is $7 \mathrm{~m} / \mathrm{s}$ in this calculation. Calculated results of the moments are shown in Figure 17.

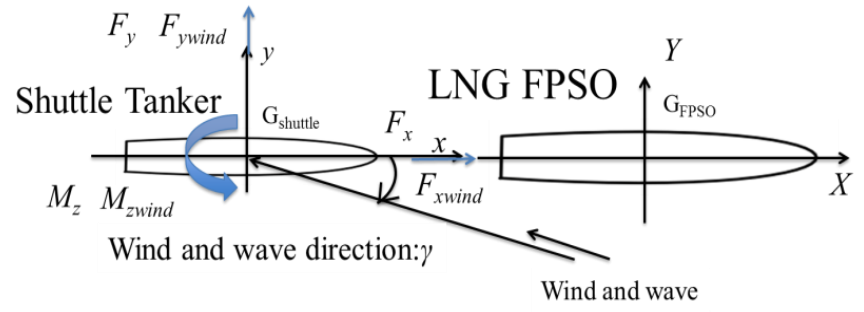

Figure 16 Coordinate systems for the calculation of the drift forces and the drift moments

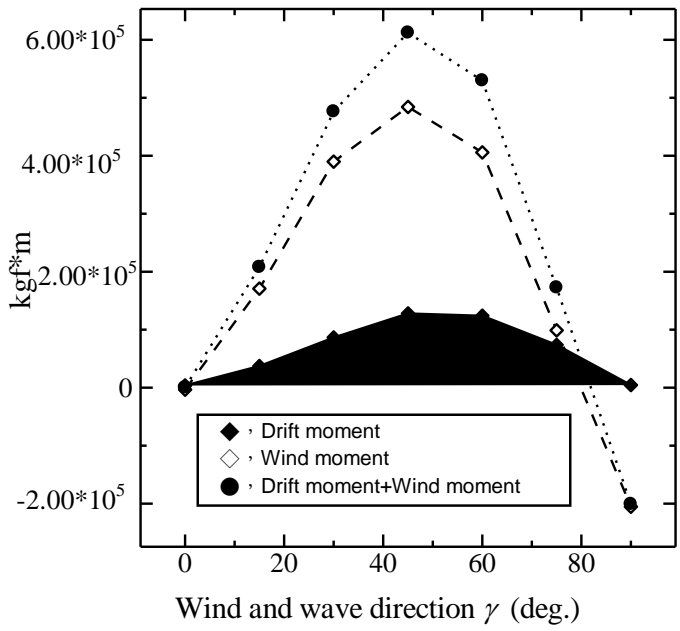

Figure 17 Calculated results of the moments acting on the shuttle tanker

As shown in Figure 17, the wave drift moments and the wind moments acting on the shuttle tanker are positive within a range from 0 degree to about 80 degrees. It is revealed that wind moments might increase amplitude of yawing motion from this figure.

The previous research about the motion characteristics of oil tankers moored by single point moorings in the shallow waters (Takarada, Obokata, 1979) revealsthat the wind forces and the wind moments decrease slewing motion generated by current forces on the lower hulls in some conditions. Also, it is revealed that the wave drift forces and the wave drift moments do not affect slewing motion of an oil tanker. Because issues of the wave drift forces and the wave drift moments are much smaller than that of wind forces and current forces when the oil tanker is moored by single point moorings. The reason why wind forces decrease the slewing motion is that a large structure on an upper deck, especially an accommodation, is located sternward. That is the point of application of the wind moment is located sternward from midship.

In the case of the shuttle tanker, however, the point of application of wind moment is not always located sternward from midship. Becauselarge LNG tank might be located forward of the ship, and the wind shielding effect might displace the point of application of wind moments. For the calculated results in Figure 17 , it is revealed that wind moments do not always decrease amplitude of the slewing motion.

In the future, we would like to study time-domain analysis of drift motion of the shuttle tanker in case that the drift force, the wind force and the mooring lines act on the shuttle tanker using Equations 16-18 in APPRENDIX and to study time-domain analysis of coupled drift motion of two floating bodies in wind

\subsection{CONCLUSIONS}

Following conclusions are obtained as follows:

1) The experiments on the ship motions of the LNG FPSO and the shuttle tanker in regular waves were conducted. In the experiments, the shuttle LNG tanker yawed drastically.

2) Mean wave drift forces and mean wave drift moments in regular waves were calculated by means of Hybrid method. As a result, it was revealed that the mean wave drift moments acting on the shuttle tanker generate yawing motion when a bow of the shuttle tanker yawed relative to the wave direction.

3) In order to confirmcharacteristics of wind effects acting on the shuttle tanker under wind shielding effect, we measured wind forces and wind moment acting on the shuttle tanker. From experimental results, it was revealed that the point of application of wind moment was located forward from midship of the shuttle tanker.

4) As simple method to estimate drift motion of the shuttle tanker in wind, wave drift forces and wind forces acting on the shuttle tanker were investigated. As a result, wind forces and wind moments might increase amplitude of yawing motion generated by wave drift forces and wave drift moments.

In the future, we would like to study time-domain analysis of the drift motion of the shuttle tanker in case that the drift force, wind force and the mooring lines act on the shuttle tanker and to study time-domain analysis of coupled drift motion of two floating bodies in wind.

\section{References}

[1] Newman, J. N. 1967. The Drift Force and Moment on Ships in Waves. Journal of Ship reseach. 11(1).

[2] Maruo, H. 1960.The Drift of a Body Floating on Waves. Journal of Ship Reseach. 4

[3] Yasunori Nihei, Takeshi Kinoshita, WeiguangBao. 2008. Nonlinear Hydrodynamic Forces on an Oscillating Floating Body in Waves-Report 1-Journal of the Japan Society of Naval Architects and Ocean Engineers 225-237. (In Japanese).

[4] ThekansaiSocietyof Naval Architects. 1983. Zosen sekkeibinran. 943. (In Japanese)

[5] NaonosukeTakarada, Jun Obokata. 1979. On the Horizontal Slow Oscillation of Ships Moored to Single Point Moorings. Journal of Naval Architects of Japan. 595: 20-28. (In Japanese). 


\section{Appendix}

Equations of drift motion of the shuttle tanker are expressed in Equations 16-18. The surge, sway, and yawing motion are taken into account in these equations. Other motions are not considered.

As shown in Appendix 1, we used a z-up moving system coordinates with their origin at a center of gravity of the shuttle tanker. Shuttle tanker is moored by the single point mooring. Where, $m, m_{x}, m_{y}, I_{z z}$ and $i_{z z}$ are mass of the shuttle tanker, added mass in $x$-direction, added mass in $y$-direction, inertia moment of the shuttle tanker and added inertia moment. $k l, F_{D x}, F_{D y} M_{D}$ are a spring tension of a mooring, drift force in $x$-direction, drift force in $y$-direction and drift moment each other. $F_{x w i n d}, F_{y w i n d}$ and $M_{z \text { wind }}$ are wind forces in $x$-direction, wind forcesb in $y$-direction and wind moment. $u, v, r$, and $L s$ also express velocity in the $x$-direction velocity in the $y$-direction and the length between a bow and center of gravity of the shuttle tanker each other. These drift forces and drift moments are changed in chronological order. A time series of the drift motions of the shuttle tanker in wind can be given by solving these equations.

$$
\begin{aligned}
& \left(m+m_{x}\right) \dot{u}-\left(m+m_{y}\right) v r=F_{x}+F_{x w i n d}+k l \cos \mu \\
& \left(m+m_{y}\right) \dot{v}+\left(m+m_{x}\right) u r=F_{y}+F_{y w i n d}+k l \sin \mu
\end{aligned}
$$

$$
\left(I_{z z}+i_{z z}\right) \dot{r}=M_{z}+M_{z w i n d}+k l L_{s} \sin \mu
$$

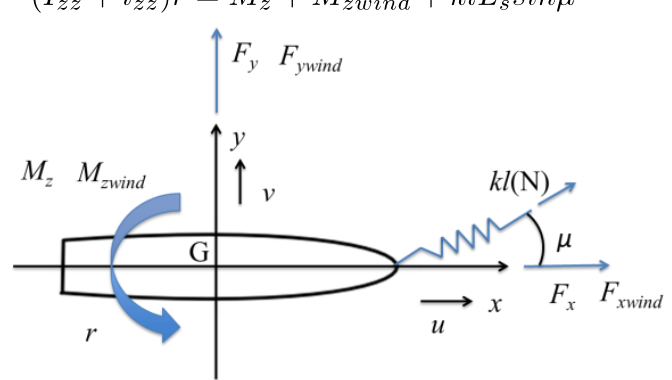

Appendix 1 Coordinate systems on the shuttle tanker 